\title{
Erratum to: Expression and significance of miRNA-21 and BTG2 in lung cancer
}

\author{
Qing Sun • Meng Hang • Xuedan Guo • Wenlong Shao • \\ Guangqiao Zeng
}

Published online: 7 June 2014

(C) International Society of Oncology and BioMarkers (ISOBM) 2014

Erratum to: Tumor Biol. (2013) 34:4017-4026

DOI 10.1007/s13277-013-0992-8

Unfortunately, the wrong Fig. 8 was included in the article.

The correct figure is the one shown below.

The online version of the original article can be found at http://dx.doi.org/ 10.1007/s13277-013-0992-8.

Q. Sun $(\bowtie) \cdot$ M. Hang $\cdot$ X. Guo

Department of Medical Oncology, Wuxi 2nd People's Hospital, Nanjing Medical University, Wuxi, China

e-mail: sunqingwx@163.com

W. Shao

Department of Cardiothoracic Surgery, The First Affiliated

Hospital of Guangzhou Medical University, Guangzhou, China

W. Shao $\cdot$ G. Zeng $(\bowtie)$

State Key Laboratory of Respiratory Disease, The First Affiliated

Hospital of Guangzhou Medical University, 151 Yanjiang Road,

Guangzhou 510120, China

e-mail: zenggqgz@yeah.net 


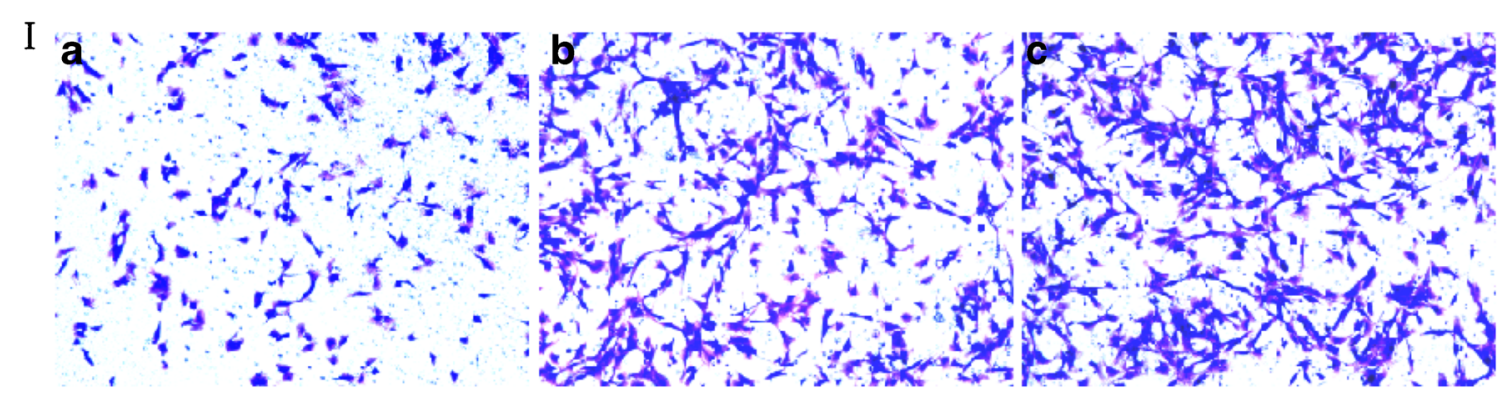

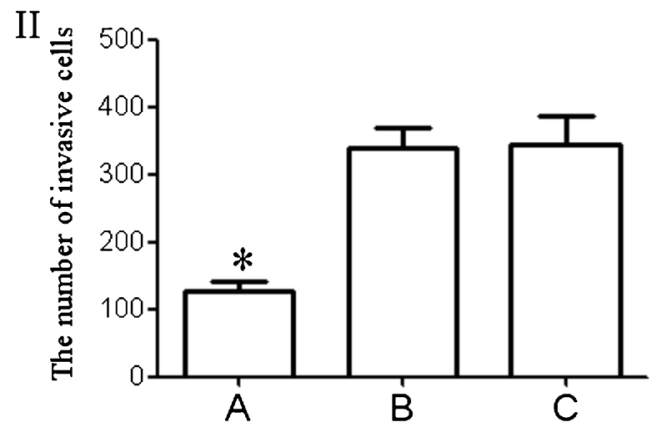

Fig. 8 Effect of miRNA-21 on invasion of 95-D cells. $I$ Crystal violet staining of 95-D cells that crossed the polycarbonate membrane of the Transwell invasion chamber, II number of cells that crossed the Transwell

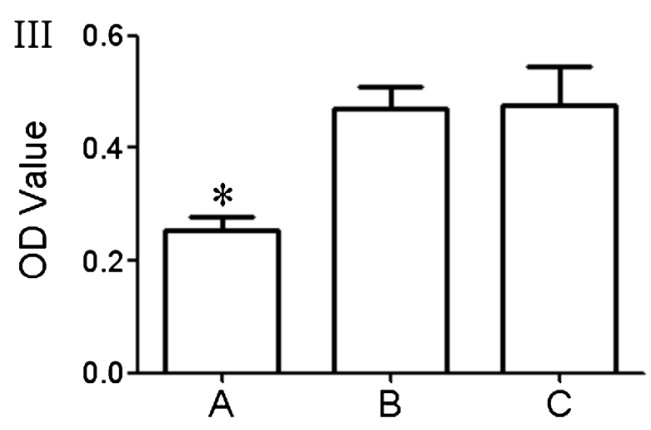

invasion chamber, $I I I$ the OD values of $95-\mathrm{D}$ cells that crossed the Transwell invasion chamber. $A$ miRNA-21 ASO-transfected group, $B$ control ASO-transfected group, and $C$ untransfected group; ${ }^{*} p<0.05$ 